\title{
Efectos de la suplementación de carnitina sobre el nivel de fatiga muscular del gastrocnemio de ratas entrenadas $y$ sedentarias
}

\section{Effect of carnitine supplementation on fatigue level in the gastrocnemius muscle of trained and sedentary rats}

\author{
Rossana Gomez Campos \\ Miguel de Arruda \\ Fabio Pinhero Ramos ${ }^{2}$ \\ Marco Antonio Cossio-Bolaños ${ }^{1}$
}

Resumo - La L-carnitina tiene un gran valor en procesos metabólicos al cumplir un papel importante en el proceso de $\beta$-oxidación mitocondrial, por lo cual, podría ser usada para mejorar el rendimiento atlético y mantener una carga de trabajo. Este trabajo tuvo por objetivo evaluar los efectos de la suplementación de L-carnitina y del entrenamiento en el tiempo necesario a la inducción de la fatiga muscular en preparaciones in situ del nervio ciático y músculo gastronemio en ratas. Cuatro grupos de animales fueron constituidos: sedentario no suplementado (SNS), sedentario suplementado (SS), entrenado no suplementado (ENS) y entrenado suplementado (ES). El entrenamiento fue realizado en sesiones diarias (1h/5dias/semana) y con suplementación oral crónica de L-Carnitina $(1 \mathrm{mg} / \mathrm{ml})$ por 4 semanas. La fatiga muscular fue obtenida mediante estimulación supramaximal tetánica del nervio ciático $(50 \mathrm{~Hz})$. Los valores del tiempo para la reducción de fuerza fueron significativamente diferentes $(\mathrm{p}<0,05)$ entre los grupos SNS vs SS y ENS vs ES, respectivamente. Sin embargo, no hubo diferencias significativas entre los grupos ES vs SS y ENS vs SNS. Estos resultados demuestran que la L-carnitina prolongo el tiempo necesario para la reducción de fuerza a nivel del músculo gastrocnemio.

Palavras-chave: Entrenamiento; Fatiga muscular; Suplementación.

Abstract - L-carnitine, considered to be of great value in metabolic processes, plays an important role in the mitochondrial $\beta$-oxidation process. It may be used to improve athletic performance and to maintain a higher workload during exercise. This study aimed to investigate the effect of L-carnitine supplementation on muscle fatigue in sciatic nerve-gastrocnemius muscle preparations in sedentary and trained rats. The animals were divided into 4 groups: non-supplemented sedentary (NSS), supplemented sedentary (SS), non-supplemented trained (NST), and supplemented trained (ST) rats. The animals were trained in daily 1-h sessions (5 days/week) and received chronic oral L-carnitine supplementation $(1 \mathrm{mg} / \mathrm{mL})$ for 4 weeks. Muscle fatigue was determined by supramaximal tetanic stimulation of the sciatic nerve (50 $\mathrm{Hz}$ ). Time values for strength reduction were significantly different $(p<0.05)$ between NSS vs. SS and NST vs. ST rats. No significant differences were observed between SS vs. ST and NST vs. NSS rats. These findings demonstrate that $L$-carnitine lengthen the time required for induction of muscle fatigue.

Key words: Muscle fatigue; Supplementation; Training.
1 Universidad Estadual de Campinas. Facultad de Educación Física. Campinas, SP. Brasil

2 Universidad Trás-os-Montes e Alto Douro. Facultad de Educación Física. Vila Real. Portugal

Recibido en 18-04-2011 Aceptado en 21-12-2011 


\section{INTRODUCCIÓN}

En la actualidad uno de los grandes problemas de la actividad física y del entrenamiento deportivo es mejorar el desempeño físico ${ }^{1}$ y combatir la fatiga muscular. Este fenómeno es considerado como uno de los tópicos principales dentro de la investigación en fisiología del ejercicio ${ }^{2}$, es decir, como un problema complejo que depende de muchos factores como el tipo, duración e intensidad del esfuerzo, tipo y densidad de las miofibrillas musculares, niveles de aptitud física individual, motivación, alimentación y condiciones ambientales ${ }^{3}$. Sin embargo, varios autores indican que la fatiga se manifiesta por la disminución de la fuerza muscular generada durante y después del ejercicio sub-máximo y máximo, así como también a la incapacidad de mantener una determinada intensidad de ejercicio en el tiempo, a la disminución de la velocidad de contracción y al aumento del tiempo de relajamiento muscular ${ }^{4}$. En este sentido, trae como consecuencia la disminución de la capacidad funcional de mantener, o continuar el rendimiento esperado ${ }^{5}$, constituyéndose de esta forma en una limitante del rendimiento físico ${ }^{3,6}$.

Por otro lado, las investigaciones actualmente están orientadas a mejorar el rendimiento físico y disminuir el fenómeno de la fatiga, demostrando algunos estudios que la suplementación nutricional ${ }^{1}$, tanto en atletas y no atletas, buscan mejorar el rendimiento deportivo, la salud y perfeccionar la forma física ${ }^{7}$. De esa forma, el uso de aminoácidos ha sido objeto de estudio por varios investigadores ${ }^{8}$, entre los que se destaca la carnitina (amina cuaternaria) por su gran valor en procesos metabólicos, actuando como cofactor en el transporte de las cadenas largas de ácidos grasos para el interior de la membrana mitocondrial con el propósito de producir energía, por lo que, se le asocia a la mejora del rendimiento deportivo en sujetos saludables por varios mecanismos, como el incremento de la oxidación de ácidos grasos, alteración de la homeostasis de la glucosa, mejoramiento de la producción de acilcarnitina y retardo en la aparición de la fatiga muscular.

En consecuencia, la carnitina juega un papel importante en el proceso de $\beta$-oxidación mitocondrial ${ }^{10}$, pudiendo ser usada para incrementar el rendimiento atlético y mantener una carga determinada de trabajo ${ }^{10}$; pero ese efecto sobre la transmisión neuromuscular aún no fue muy investigado $^{11}$, inclusive se sabe muy poco en relación al efecto del entrenamiento sobre el contenido de L-carnitina en las células musculares ${ }^{12}$ y merece ser estudiado. Por lo tanto, el objetivo del presente estudio fue determinar el efecto de la suplementación de L-carnitina sobre el nivel de fatiga muscular del músculo gastrocnemio de ratas sedentarias y entrenadas.

\section{MATERIAL Y MÉTODOS}

\section{Muestra y tipo de investigación}

El estudio es de tipo experimental ${ }^{13}$, donde fueron seleccionados de manera probabilística aleatoria ratas jóvenes adultos machos de raza Wistar, cuyo 
peso corporal osciló entre 350 a 380 gramos. Los animales provienen del Bioterio del Departamento de Farmacología de la UNICAMP. Los animales fueron alojados en cajas colectivas con cinco animales en cada una, en ciclos de claro/oscuridad (12/12 h), recibiendo ración patrón (Labina, Purina) y agua ad libitum.

Por otro lado, fueron constituidos 4 grupos de animales $(n=5)$ : sedentario suplementado con L-carnitina (SS), sedentario no suplementado (SNS), entrenado suplementado con L-carnitina (ES) y entrenado no suplementado (ENS). Estos cuatro grupos obedecen a un diseño experimental de Solomon ${ }^{14}$. El estudio fue aprobado por el Comité de Ética de la Facultad de Biología de la UNICAMP, Brasil.

\section{Evaluación del peso corporal}

Para la evaluación del peso corporal ( $\mathrm{g}$ ) de ratos machos se utilizó una balanza analítica de marca Scaltec modelo SAC-62, con una precisión de $\left(10^{-4}\right.$ gramos), siguiendo las recomendaciones de Cossio-Bolanos, et.al ${ }^{15}$. El procedimiento consistió en colocar a los animales en un frasco ligero sobre la balanza con el objetivo de evaluar el peso corporal en gramos (g). Le evaluación fue repetida dos veces, con la intención de verificar el error técnico de la medida ETM intra-evaluador $\left(\sqrt{d}^{2} / 2 n\right)$, así también se determinó la reproductibilidad del peso corporal a través del coeficiente intraclase $(\mathrm{r}=0,99)$.

\section{Suplementación}

Los animales fueron suplementados vía oral, con una solución conteniendo aproximadamente $1 \mathrm{mg} / \mathrm{ml}$ de L-carnitina ${ }^{16}$, equivalente a una dosis aproximada de $0.2 \mathrm{~g} / \mathrm{kg}$ por día. Esta dosis representa aproximadamente el doble del consumo diario de L-carnitina ${ }^{17}$ y mantiene los niveles plasmáticos de carnitina en aproximadamente 3 veces por encima de los niveles observados en animales control.

\section{Entrenamientos de las ratas}

Las sesiones de entrenamiento fueron realizadas en una cinta rodante eléctrica (banda sin fin), con carriles individuales. En la primera semana del estudio, todos los animales fueron sometidos a un período de adaptación a la cinta rodante, en el que corrieron a una velocidad que varió entre $5 \mathrm{~m} /$ min en el primer día, hasta $10 \mathrm{~m} / \mathrm{min}$ en el quinto día de la semana, aumentando progresivamente el tiempo en la duración de las sesiones, hasta que los animales consiguieran permanecer corriendo en la cinta rodante por 60 minutos de forma continua.

Después de la adaptación se inicio el periodo de entrenamiento físico que fue compuesto por sesiones diarias de corrida en la banda sin fin, con una duración de 60 minutos, cinco días por semana durante cuatro semanas. La velocidad de la cinta rodante fue aumentándose progresivamente conforme la evolución del grupo de animales hasta alcanzar la velocidad final de $25 \mathrm{~m} / \mathrm{min}$. Este protocolo de entrenamiento 
implica la realización de ejercicio aeróbico, propuesto para ratas, como lo describe Manchado, et.al ${ }^{18}$.

Finalmente, al término del periodo total de entrenamiento, todos los animales se mantuvieron en reposo por un período de 48 horas antes de ser sacrificados.

\section{Evaluación de la fuerza de contracción muscular}

Para la determinación de la fuerza de contracción muscular, los animales fueron anestesiados con uretana (1,2 g/Kg, solución al 2\%, i.p), administrada por vía peritoneal. Después de la anestesia, fueron colocados en decúbito ventral para iniciar el proceso de disección del músculo gastrocnemio y nervio ciático. El tendón calcáneo del músculo gastrocnemio fue fijado por medio de un hilo quirúrgico a un transductor de fuerza. El registro de la fatiga se llevó a cabo a través del sistema PowerLab 400 de adquisición de datos (software versión 5.0, AD-Instruments, Colorado Springs, EUA). Durante la realización de los experimentos el nervio ciático y músculo fueron hidratados constantemente por goteamiento de solución salina ( $\mathrm{NaCl} 0,9 \%)$.

Para cada preparación fue realizada la estimulación indirecta (vía nervio) del músculo, colocando los electrodos de estimulación en contacto con el nervio ciático. Inicialmente fue realizada una estimulación eléctrica supra-umbral del nervio por un período de 3 a 4 minutos con pulsos cuadrados de voltaje, con frecuencia de $0,5 \mathrm{~Hz}$, con duración de $1 \mathrm{~ms}$. Esto con la finalidad de estabilizar los potenciales de acción (avalos). Luego, fue realizada la tetanización del músculo mediante el incremento de la frecuencia del estímulo para $50 \mathrm{~Hz}$. El estímulo se mantuvo hasta que la fuerza de contracción decrezca hasta los niveles basales.

\section{Análisis estadístico}

Los resultados del presente estudio fueron analizados a través de la estadística descriptiva de la media $(\mathrm{X})$ y desviación estándar (DE) y para determinar las diferencias estadísticas entre los grupos se utilizó el análisis de varianza (ANOVA, de dos vías) y la prueba de especificidad de Bonferroni. En todos los casos se adopto un nivel de significancia de $\mathrm{p}<0,05$. La distribución normal de los valores fue verificada a través del test de Kolmogorov-Smirnov, verificándose distribución normal.

\section{RESULTADOS}

El error técnico ETM intra-evaluador del peso (g) de las ratas de los 4 grupos muestra valores entre 0,55 a $0,94 \%$, siendo aceptables estadísticamente. La reproducibilidad de las medidas de la masa corporal ha resultado ser altamente reproductible, según lo reflejado en los valores del coeficiente de correlación intra-clase (CCI), cuyos valores oscilaron entre $0,98-0,99$, respectivamente. 
La tabla 1 muestra las características de la población en valores medios (X) y desviación estándar (DE) del peso corporal (g) y del peso del músculos gastrocnemio $(\mathrm{g})$. Esos valores no muestran diferencias significativas cuando fueron comparadas entre grupos de las mismas condiciones (SS vs SNS) y (ES vs ENS).

Tabla 1. Caracterización de la población estudiada $(n=20)$.

\begin{tabular}{lcccc}
\hline Grupos & SNS & SS & TNS & TS \\
\hline N & 10 & 10 & 5 & 5 \\
Peso inicial (g) & $353 \pm 12$ & $353 \pm 12$ & $358 \pm 14$ & $356 \pm 17$ \\
Peso final (g) & $456 \pm 7^{\mathrm{a}}$ & $435 \pm 9^{\mathrm{a}}$ & $375 \pm 17^{\mathrm{bc}}$ & $401 \pm 9^{\mathrm{abc}}$ \\
Ganancia de peso corporal (g) & $103 \pm 13$ & $83 \pm 12$ & $17 \pm 12^{\mathrm{bc}}$ & $45 \pm 15^{\mathrm{b}}$ \\
Peso del músculo gastrocnemio (g) & $3,0 \pm 0.1$ & $3,2 \pm 0.1$ & $2,7 \pm 0.2$ & $3,1 \pm 0.2$ \\
\% del peso corporal & 0.66 & 0,74 & 0,71 & 0,77 \\
\hline
\end{tabular}

${ }^{(a, b, c)} p<0,05$ (significativo). a. Indica diferencia estadística entre el peso inicial y final intragrupo, b. Indica diferencia estadística en relación al grupo SNS, c. Indica diferencia estadística en relación al grupo SS

En relación a las comparaciones de los pesos corporales de las ratas realizadas al inicio y al final (intra-grupo), se verificó la existencia de diferencias estadísticamente significativas, resaltando que en el grupo (SS) aumentó el peso en un 15,06\% producto de la suplementación y el grupo (SNS) aumentó en un 17,60\%. Ese aumento en el porcentaje se deba probablemente a la inactividad física (sedentarismo) producido durante las 4 semanas durante el experimento.

Por otro lado, en relación a las comparaciones del peso final inter-grupos se observó que entre los grupos (SS vs SNS) y (ES vs ENS) no existe diferencias significativas. Sin embargo, se verificó diferencias entre los grupos (SS vs ES) y (SNS vs ENS). Esos resultados permiten afirmar que el entrenamiento y la suplementación ayudan a mantener relativamente estable el peso corporal. Cabe destacar que en el presente estudio no se evalúo la composición corporal de las ratas, ya que podrían explicar mejor ese efecto a partir de los componentes corporales (grasa, músculo, tejido óseo y residual).

La tabla 2, figura 1, muestra los resultados de la reducción de fuerza $(\mathrm{mN})$ durante el tiempo (minutos), cuando se comparó todos los grupos, observándose que el grupo (SNS) fatigó a los 41 minutos y el grupo TNS a los 49 minutos, teniendo una diferencia de 8 minutos, el grupo (SS) demoró en fatigar 81 minutos y el grupo (TS) llegó a los 87 minutos. Estos resultados permiten destacar que el entrenamiento no fue capaz de aumentar el tiempo de reducción de la fuerza (fatiga).

Por otro lado, estos resultados indican que la suplementación de carnitina aumentó el tiempo necesario para soportar la fatiga durante el tiempo, mejorando en 40 min (SNS vs SS) y 38 minutos (TNS vs TS), respectivamente.

Finalmente, estos resultados indican que el entrenamiento junto con la suplementación prolongan aún mas el tiempo de reducción de la fuerza durante el tiempo. 
Tabla 2. Valores de la reducción de fuerza $(\mathrm{mN})$ durante el tiempo ( $\mathrm{min})$ de ratas sedentarias suplementadas (SS), sedentarias no suplementadas (SNS), entrenadas suplementadas (ES) y entrenadas no suplementadas (ENS).

\begin{tabular}{|c|c|c|c|c|c|c|c|c|}
\hline \multirow{2}{*}{ Tiempo (min) } & \multicolumn{2}{|c|}{ SNS } & \multicolumn{2}{|c|}{ SS } & \multicolumn{2}{|c|}{ TNS } & \multicolumn{2}{|l|}{ TS } \\
\hline & $X \pm D E$ & $\%$ & $X \pm D E$ & $\%$ & $X \pm D E$ & $\%$ & $X \pm D E$ & $\%$ \\
\hline 0 & $37.1 \pm 3.4$ & 100 & $41.1 \pm 3.3$ & 100 & $40.9 \pm 2.3$ & 100 & $46.3 \pm 1.0$ & 100 \\
\hline 1 & $13.1 \pm 1.9$ & 35,2 & $23.9 \pm 1.5^{\mathrm{a}}$ & 59,8 & $19.0 \pm 3.7$ & 46,2 & $34.2 \pm 5.5^{\mathrm{abc}}$ & 73,6 \\
\hline 5 & $8.4 \pm 1.0$ & 22,6 & $15.9 \pm 1.5$ & 38,8 & $14.0 \pm 3.2$ & 34,1 & $23.2 \pm 2.7^{a b c}$ & 49,9 \\
\hline 10 & $5.6 \pm 1.4$ & 15,4 & $11.0 \pm 2.1^{\mathrm{a}}$ & 26,2 & $11.7 \pm 2.9^{a}$ & 28,5 & $18.9 \pm 2.0^{\mathrm{abc}}$ & 40,8 \\
\hline 15 & $4.2 \pm 1.4$ & 11,9 & $7.9 \pm 2.7$ & 18,1 & $10.1 \pm 2.9$ & 24,5 & $16.5 \pm 1.5^{\mathrm{ab}}$ & 35,7 \\
\hline 20 & $3.2 \pm 1.4$ & 9,2 & $5.7 \pm 3.1$ & 12,9 & $8.2 \pm 2.5$ & 20,1 & $14.2 \pm 1.5^{\mathrm{ab}}$ & 30,6 \\
\hline 25 & $2.0 \pm 1.2$ & 5,6 & $5.1 \pm 2.9$ & 11,5 & $6.0 \pm 1.6$ & 15,1 & $12.4 \pm 1.9 \mathrm{abc}$ & 26,8 \\
\hline 30 & $1.6 \pm 1.0$ & 4,6 & $4.4 \pm 2.7$ & 9,8 & $4.6 \pm 1.2$ & 11,5 & $10.2 \pm 2.4^{a}$ & 22,2 \\
\hline 35 & $0.9 \pm 0.6$ & 2,7 & $3.6 \pm 2.5$ & 8,1 & $1.9 \pm 1.2$ & 5,3 & $8.4 \pm 2.5$ & 18,3 \\
\hline 40 & $0.1 \pm 0.1$ & 0,2 & $2.5 \pm 2.1$ & 5,3 & $1.0 \pm 1.0$ & 3,2 & $5.9 \pm 2.9$ & 13,1 \\
\hline 45 & 0 & 0 & $1.8 \pm 1.8$ & 3,8 & $0.9 \pm 0.9$ & 2,7 & $5.4 \pm 2.8$ & 11,8 \\
\hline 50 & & & $1.7 \pm 1.7$ & 3,5 & 0 & 0 & $4.0 \pm 2.1$ & 8,8 \\
\hline 55 & & & $1.2 \pm 1.2$ & 2,6 & & & $3.0 \pm 1.8$ & 6,6 \\
\hline 60 & & & $1.0 \pm 1.00$ & 2,1 & & & $2.5 \pm 1.5$ & 5,2 \\
\hline 65 & & & $0.8 \pm 0.8$ & 1,6 & & & $1.8 \pm 1.2$ & 3,9 \\
\hline 70 & & & $0.5 \pm 0.5$ & 1,0 & & & $1.2 \pm 1.0$ & 2,6 \\
\hline 75 & & & $0.3 \pm 0.3$ & 0,6 & & & $0.9 \pm 0.8$ & 2.0 \\
\hline 80 & & & $0.2 \pm 0.2$ & 0,3 & & & $0.5 \pm 0.5$ & 1,2 \\
\hline 85 & & & 0 & 0 & & & $0.2 \pm 0.2$ & 0,4 \\
\hline
\end{tabular}

${ }^{(a, b, c)} \mathrm{p}<0,05$ (significativo). a. Indica diferencia estadística en relación al grupo SNS, b. Indica diferencia estadística en relación al grupo SS, c. Indica diferencia estadística en relación al grupo TNS.

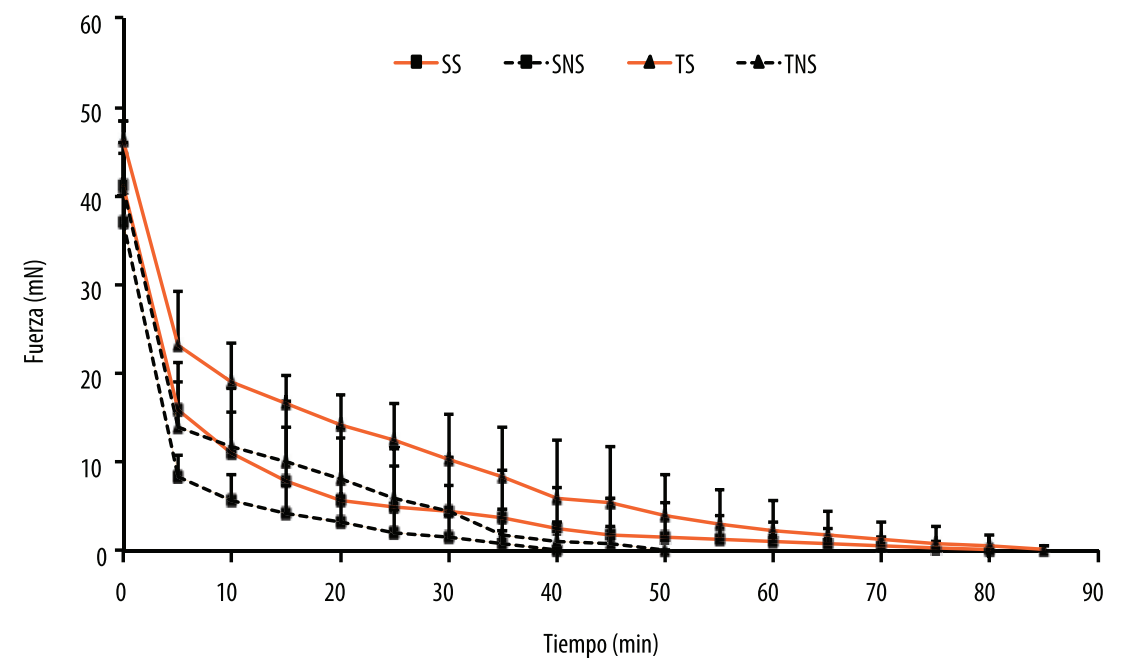

Figura 1. Reducción de la fuerza en el tiempo de los grupos sedentario no suplementado (SNS), sedentario suplementado (SS), entrenado no suplementado (ENS) y entrenado suplementado (ES). 


\section{DISCUSIÓN}

La suplementación de la carnitina ha planteado la hipótesis de mejorar el rendimiento del ejercicio en personas sanas a través de diversos mecanismos, incluyendo mejoras en la oxidación de ácidos grasos del músculo, alteraciones en la homeostasis de la glucosa, mejoramiento de la producción de acilcarnitina, modificación de la respuesta al entrenamiento y alteraciones en la resistencia a la fatiga muscular ${ }^{9}$. En este sentido, teniendo en cuenta su efecto sobre el metabolismo oxidativo, en el presente estudio se constató que la suplementación de L-carnitina prolongó el tiempo de la reducción de la fuerza (fatiga) del músculo gastrocnemio en ratas entrenadas suplementadas (43\%) y ratas sedentarias suplementadas (48\%), cuando fueron inducidas por estimulación eléctrica in vivo hasta la exhaustación, llegando a fatigar el grupo entrenado suplementado (ES) a los 88 minutos y el grupo sedentario suplementado (SS) a los 82 minutos, respectivamente.

Estas evidencias fueron constatadas por otros estudios en modelos animales ${ }^{12,19,20}$ en el que hacen suponer que el efecto se deba al aumento de la tasa de oxidación de los ácidos grasos de cadena larga, permitiendo el almacén de glucógeno, produciéndose por ende, mayor producción de ATP durante el ejercicio ${ }^{21}$. Tales resultados se apoyan en los postulados realizados por Wagenmakers ${ }^{22}$ en el que sugiere que la carnitina presentaría un efecto ergogénico, inclusive Gorostiaga et $\mathrm{al}^{23}$ considera que la suplementación de la L-carnitina podría aumentar la oxidación de los ácidos grasos, lo que aumentaría el ATP disponible para la realización del trabajo mecánico. Sin embargo, algunos autores investigando en modelos humanos concluyen que no tuvieron efectos positivos sobre el rendimiento atlético ${ }^{19,24}$, contradiciendo de esta forma los resultados del presente estudio.

De hecho, algunos autores como Lancha, et.al ${ }^{8}$ y Wyss, et.al ${ }^{25}$ consideran que la suplementación de la L-carnitina en ratas entrenadas por medio de ejercicios de larga duración retardan la fatiga muscular, así como también, se sugiere el uso para la prevención del daño de los músculos esqueléticos $^{26}$, aumento de la regulación del flujo sanguíneo y la distribución de $\mathrm{O}_{2}$ para el tejido muscular durante y después del ejercicio físico ${ }^{27}$, respectivamente.

Desde esa perspectiva, se considera como posibles limitaciones dentro del estudio la ausencia de ensayos clínicos adicionales, lo que hubiera permitido aclarar los resultados del presente estudio. A su vez, el tamaño de la muestra en el grupo de los animales entrenados es inferior en relación a los animales sedentarios, lo que podría producir un ligero sesgo en los resultados.

Finalmente, respecto a la aplicabilidad de la suplementación de la Lcarnitina, algunos estudios sugieren que podría actuar en la reducción de calambres y el aumento de la sensación de bienestar y calidad de vida ${ }^{27}$, así como en el tratamiento del síndrome de la fatiga crónica ${ }^{28}$. Por lo tanto, se sugiere que futuros estudios deban incluir grupos de placebo en los experimentos, variables como el $\mathrm{VO}_{2}$ máximo, umbral de lactato, así como 
la necesidad de considerar el grado de crecimiento y maduración de los animales a estudiar

\section{CONCLUSIÓN}

A través de los resultados obtenidos, se puede concluir que la suplementación en animales sedentarios y entrenados con L-carnitina aumentó el tiempo necesario para la reducción de la fuerza (fatiga) a nivel del músculo gastrocnemio, sugiriendo que la L-carnitina desempeña un importante papel en el proceso de contracción muscular y por ende, mejora el desempeño del trabajo prolongado.

\section{REFERENCIAS BIBLIOGRÁFICAS}

1. Pombo R, César C, Dellú A, Lima E, Cusma N, Souza R, et.al. Efeitos da suplementação aguda de aspartato de arginina na fadiga muscular em voluntários treinados. Rev Bras Med Esporte 2005;11(6):347-351.

2. Ascensão A, Magalhães J, Oliveira J, Duarte J, Soares. Fisiologia da fadiga muscular. Delimitação conceptual, modelos de estudo e mecanismos da fadiga de origem central e periférica. Rev Port Ciên Desp 2003;3(1):108-23.

3. Fitts RH. Mecanismos de fadiga muscular. In: Prova de esforço e prescrição de exercício. American College of Sports Medicine. São Paulo: Revinter 1994;73-79.

4. Bangsbo J. Physiology of muscle fatigue during intense exercise. In. Reilly T, Orme M, editors. The clinical pharmacology of sport and exercise, Elsevier Science BV 1997; p.123-130.

5. Rossi L, Tirapegui J. Aspectos atuais sobre exercício físico, fadiga e nutrição. Rev Paul Educ Fís 1999;13:67-82.

6. Gibson H, Edwards RHT. Muscular exercise and fatigue. Sports Med 1985;2:120-32.

7. Gomes M, Tirapegui J. Relação de alguns suplementos nutricionais e o desempheno físico. Arch Latinoam Nutr 2000; 50(4):317-29

8. Lancha Jr AH, Recco MB, Abdalla DSP, Curi R. Effects of aspartate, asparagine and carnitine supplementation in the dieto $\mathrm{n}$ metabolims of skeletal muscle during a moderate exercise. Physiol Behav 1995; 57(2):367-71.

9. Brass EP. Supplemental carnitine and exercície. Am J Clin Nutr 2000;72(2):612-23.

10. Swart L, BSc(Hons), Rossouw J, Loots JM, Kruger MC. The effect of L-carnitine supplementation on plasma carnitine levels and various performance parameters of male marathon athletes. Nutr Res 1997;17(3):405-14.

11. Lopez G, Bazotte RB, Curi R, Alves-Do-Prado W. L-and DL-carnitine induce titanic fade in rat neuromuscular preparation. Braz J Med Biol Res 2003; 36(9):1255-62.

12. Bacurau RFD, Navarro F, Bassit R.A, Meneguello MA, Santos VT, Almeida ALR, et.al. Does exercise training interfere with the effects of l-carnitine supplementation. Nutrition 2003;19:337-41.

13. Thomas J, Nelson J. Research Methods in Physical Activity. Human Kinetics, 1996.

14. Campbell DT, Stanley JC. Diseños experimentales y cuasiexperimentales en la investigación social. Buenos Aires: Amorrortu; 1973.

15. Cossio MA, Gómez R, Arruda M, Hochmuller R. Valores de confiabilidad de indicadores somáticos en ratas machos wistar. Actualizacion en Nutricion 2010;11(4):296-302.

16. Malone JI, Cuthbertson DD, Malone MA, Schocken DD. Cardio-protective effects of carnitine in streptozotocin-induced diabetic rats. Cardiovasc Diabetol 2006; 5(2):1-6.

17. Borum PR. Carnitine. Annu Rev Nutr 1983;3:233-59. 
18. Manchado FB, Gobatto CA, Contarteze RVL, Papoti M, Mello MAR. Maximal lactate steady state in running rats. J Exerc Physiol 2005;8:1-8

19. Clarkson PM. Nutrition for improved sports performance. Current issues on ergogenic aids. Sports Med 1996;2(6):393-401.

20. Brass EP, Hiatt WR. The role of carnitine supplementation during exercise in man and in individuals with special need. J Am Coll Nutr 1998;17:207-15

21. Carter AL, Abney PO, Lapp F.D. Byosintesis and metabolism of carnitine. J Child Neurol 1995;10:253-7.

22. Wagenmakers A. L-carnitine supplementation and performance in man. Med Sci Sports Exerc 1991;32:110-27.

23. 23. Gorostiaga EM, Maurer CA, Eclache JP. Decrease in respiratory quotient during exercise following L-carnitine supplementation. Int J Sports Med 1989;10:169-74.

24. Hawley JA, Brouns F, Jeukendrup A. Strategies to enhance fat utilization during exercise. Sports Med 1998;24(4):241-57.

25. Wyss V, Ganzit GP, Riezi. A. Effects of L-carnitine administration on VO2max and the aerobic-anaerobic threshold in normoxia and acute hypoxia. Eur J Appl Physiol 1990; 60:1-6.

26. Volek JS, Kraemer WJ, Rubin MR, Gómez AL, Ratamess NA, Gaynor P. L-carnitine L-tartrate supplementation favourably affects markers of recovery from exercises stress. Am J. Physiolo Endocrinol Metab 2002; 282:474-482.

27. Spagnili LG, Palmieri G, Mauriello A, Vacha GM, D lddios S, Giorcelli G, et.al. Morphometric evidence of the trophic effect of L-carnitine on human skeletal muscle. Nephron 1990;55(1):16-23.

28. Plioplys AV, Plioplys S. Amantadine and L-carnitine treatment of Chronic Fatigue Syndrome. Neuropsychobiology 1997;35:16-23.

Dirección postal

Rossana Gómez Campos Av. Erico Verissimo 701. Ciudad Universitaria

CEP. 13083-851. Campinas, SP. Brasil. E-mail: rossanagomez_c@hotmail. com 